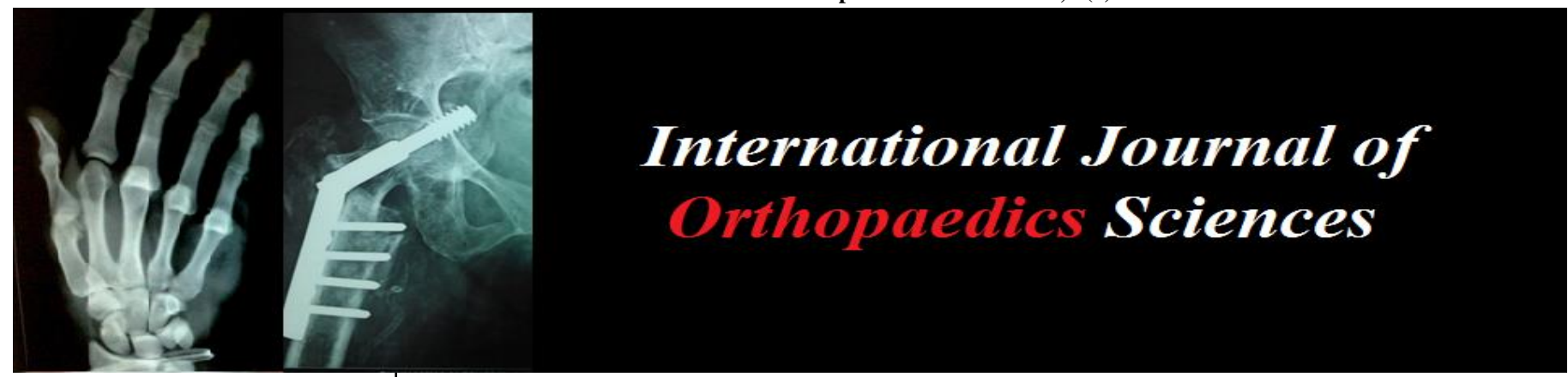

ISSN: $2395-1958$

IJOS 2018; 4(3): 145-149

(C) 2018 IJOS

www.orthopaper.com

Received: 26-05-2018

Accepted: 27-06-2018

Joe J Wilbur Gonsalves

Kanachur Institute of Medical

Sciences Mangalore University

Road, Natekal, Karnataka, India
Correspondence

Joe J Wilbur Gonsalves

Kanachur Institute of Medical Sciences Mangalore University Road, Natekal, Karnataka,

India

\section{A comparative study of locking plate by MIPO versus closed interlocking intramedullary nail in extra- articular distal tibia fractures}

\author{
Joe J Wilbur Gonsalves \\ DOI: https://doi.org/10.22271/ortho.2018.v4.i3c.26
}

\section{Abstract}

Background: Distal tibia fractures have the second highest incidence of all tibia fractures after the middle third fracture of the tibia. Management of the distal tibia fractures is the most challenging due to its superficial nature and propinquity to the ankle joint. There are various management methods of distal tibia fractures. Distal tibia fractures can be managed using closed reduction and casting or surgical interventions such as closed reduction and intramedullary nailing or open reduction and internal fixation with plating or closed reduction and percutaneous plating or external fixators.

Methods: A comparative study of locking plate by MIPO versus closed interlocking intramedullary nailing in extra-articular distal tibia fractures was undertaken among 30 patients with closed extraarticular distal tibia fractures, in the department of orthopedics, Ruby Hall Clinic, Pune from March 2014 to March 2015. The results were assessed using Johner and Wruh's criteria.

Results: 15 patients were treated with closed interlocking intramedullary nail and 15 patients with MIPO technique. The patients were operated. No difference was observed in terms of duration of surgery, time for fracture union and malunion. Difference was observed in the terms of total weight bearing after surgery, infection and other complications.

Conclusion: It is observed that interlocking nailing is better in terms of early total weight bearing, fast union, and decreased risks of complications. Thus, it is a better choice for patient undergoing the treatment for extra- articular distal tibia fractures.

Keywords: Locking plate, MIPO versus closed interlocking intramedullary nail, extra-articular distal tibia fractures

\section{Introduction}

The incidence of injuries and fractures has greatly increased in the present than the olden days. Among all kinds of fractures, tibia fracture is the most common long bone fracture experienced by the orthopedic surgeons. Distal tibia fractures have the second highest incidence of all tibia fractures after the middle third fracture of the tibia. Management of the distal tibia fractures is the most challenging due to its superficial nature and propinquity to the ankle joint. Various risks are associated with the management of such fractures, such as nonunion, osteomyelitis or delayed union ${ }^{[1]}$. There are different kinds of distal tibia fractures classified according to various surgeons. Ellis (1958) classified tibia fractures, according to the mode of injury, such as direct or indirect. Micheal Alms (1962) classified tibia fractures based on the type and site of trauma. It includes transverse, transverse oblique, spiral and comminuted. Further, it was divided into the parts of the bone- upper third, middle third and lower third. Robinson et al. classified it by the mode of injury, such as bending and torsion.

There are various management methods of distal tibia fractures. Distal tibia fractures can be managed using closed reduction and casting or surgical interventions such as closed reduction and intramedullary nailing or open reduction and internal fixation with plating or closed reduction and percutaneous plating or external fixators ${ }^{[2]}$. It demands extensive soft tissue dissection and devitalisation, prolongs fracture healing due to less favorable healing conditions, and more prone to infection and postoperative ankle stiffness ${ }^{[3-5]}$. Due to these complications other methods are being used. The two most common and effectively used techniques are closed intramedullary interlocking nail and minimally invasive plate osteosynthesis (MIPO) ${ }^{[2]}$.

$$
\sim 145 \sim
$$


The intramedullary nails provide immobility to the fractured fragments of the tibia by functioning as flexible internal splints. It acts as a load sharing tool, which is situated close to the neutral axis of the bone with minimal strain thus, reducing the stress shielding. The intramedullary nails provide three point fixation, elastic impringement and provides stability by interlocking bolts at both the proximal and distal to the fracture site. Intramedullary nails for distal tibia fracture reduces surgical injuries to the bone and the soft tissues ${ }^{[7]}$. It reduces the time of union as the nails cannot be rigid and the movement at the fracture site along with axial micro motion in dynamically locked nails promotes external bridging callus. There is also a possibility of increased periosteal blood supply and diminished medullary blood supply, which causes rapid reunion of the bone. Intramedullary nailing reduces the chances of Stress Protection Osteopenia. It minimizes the risk of re-fracture after the removal of the nail ${ }^{[6]}$.

The procedure is difficult to perform. The malalignment rates are high, ranging from $5 \%$ to $58 \%$ as the reduction of proximal and distal metaphysical fractures is complex to manage with an intramedullary device. Intramedullary nailing has been associated with knee pain in about $71 \%$ of the patients with such fractures. Intramedullary nailing is known to cause diminished blood supply to the fractured bone (8). In case of a loose fitting nail, the vascular supply is reestablished quickly, but in a well-adjusted nail the vascular supply depends on the periosteal blood supply. When the blood supply is delayed for a longer time, the soft tissues of the diaphysis gets devascularised leading to delayed union of the fractured bone and infection. MIPO is an another management for these kinds of fractures. It requires a smaller area for reduction, promoting effective biological fixation. Nevertheless, complications may include pseudoarthrosis, delayed or non-union and angular deviations ${ }^{[1]}$.

Minimally invasive plate osteosynthesis is a procedure of fracture management in which a plate is placed across a fracture through small incisions on the skin at the ends of the fractured bone. The principle of a fracture healing is stability of the fractured bone with improved vascularity. Initially, the procedure bridge plating was used and conventional plate was applied using large incisions for femoral fractures. The preservation of the vastus lateralis muscles caused healing with abundant callus formation ${ }^{[9]}$. Gradually, the size of the incisions was reduced to only 2 incisions at the end of the bone with the plates placed sub-muscularly over the fractured bone fragments. The locked plates restrict the axial orientation of the screw to the plate, improving the stability by creating a single beam construct of screw-bone-plate. In such a construct there is no movement between the screw, bone and plate. The single beam construct is stronger than load sharing beam construct as in the load sharing construct there is motion between the components. The plate is the single beam while the contruct act as a fixed angle device.The plate supports the fracture fixation when the fracture or bone cannot accept numerous screws for fixation and minimize gap strain by achieving plate bone compression ${ }^{[9,4]}$.

There are certain methods of treatment of distal tibia fractures. Closed reduction and plaster immobilisation is done for closed and stable fractures. Plaster casts with transfixation pins are used in unstable fractures. Long oblique or spiral fractures require screws for fixation. Intramedullary nailing can be used to treat tibia shaft fractures distal tibia fractures. There are a number of complications associated with the fracture of distal tibia such as non-union or delayed union of the fractured bone, malunion, shortening, infection, compartment syndrome, i.e. fracture with soft tissue injury, necrosis of the skin, joint stiffness, traumatic arthritis, reflex sympathetic dystrophy, refracture and fat embolism (7).

\section{Aim}

The aim of this study was to compare outcome of fractures of the distal tibia treated by interlocking intramedullary nails and locking plate by MIPO in terms of -

- Rate of union

- Radiological union time

- Duration of Surgery

- Functional outcome e.g. range of motion at the ankle joint etc.

- Complications such as infections, delayed/non-union, implant irritation

\section{Materials and methods}

Study design: A comparative study of "locking plate by MIPO versus closed interlocking intramedullary nailing in extra-articular distal tibia fractures" was undertaken in the department of orthopedics, Ruby Hall Clinic, Pune from March 2014 to March 2015 after obtaining ethical clearance.

Patient's enrollment: The study included both male and female patients. The patients operated after March 2014 were studied and followed prospectively.

Sample size: Thirty consecutive patients with distal tibia fractures were included in the study. Half of the patients were treated with half with closed interlocking intramedullary nailing and the other half were treated with locking plate by MIPO. All the patients had fresh fractures and were traumatic in nature. Most were the patients were admitted in the casualty while a few were admitted through the outpatient department.

The patients were randomly divided into two groups based on their method of treatment. Group A consisted of patients who were treated by closed intramedullary interlocking nail and group B consisted of patients who were treated locking plate by MIPO.

\section{Inclusion criteria}

1. Age more than 18 years.

2. Closed extra-articular distal tibia fractures within $5 \mathrm{~cm}$ of ankle joint.

3. Patients who gave consent to participate in the study and procedure.

\section{Exclusion criteria}

1. Age less than 18 years.

2. Patients refusing to provide consent to in the study and procedure.

3. Open fractures of distal tibia.

4. Intra-articular fractures of distal tibia.

5. Pathological fracture.

6. Pre-existing local skin lesions

\section{Management protocol}

1. Administration of first aid on reception of the patient in casualty department.

2. Careful assessment of injured limb as regards to side affected, extent of soft tissue injury, deformity and neurovascular status.

3. Thorough examination of the patient to rule out head/ chest/ spinal and abdominal injury. 
4. Musculoskeletal examination to rule out other associated fractures.

5. Stabilization of the patient with i.v. fluids, oxygen, and blood transfusion whenever required.

6. Primary immobilization of the injured limb in above knee plaster slab and transportation of the patient to radiology department for X-rays.

7. The patient was then admitted to respective ward and evaluated in terms of time, mode of injury, radiological assessment with anteroposterior and lateral X-rays of the limb which included proximal and distal joints.

8. Leg elevation, analgesics and inj.Tetanus $0.5 \mathrm{cc}$ i.m. were given as indicated.

In both the kinds of treatment methods, the fracture was reduced by closed method. Locking plate was done with minimally invasive techniques (MIPO).The associated fibula fracture was fixed when the fracture was lying within $10 \mathrm{cms}$ from the tip of the lateral malleolus.

\section{Data collection}

For all the patients, the alternate skin sutures were removed on the $10^{\text {th }}$ postoperative day. All the sutures were removed on the $15^{\text {th }}$ postoperative day after checking for gaping. Toe touch weight bearing with crutches/walker was initiated after 10 days depending upon the type of fracture and rigidity of fixation. Further follow-up was done at three week intervals was done for every patient. Each patient was assessed clinically and radiographically in a pre-determined proforma (Annexure A).

Follow-up was carried out at 3 weeks interval initially for the first two months. Thereafter, it was done at an interval of 6 weeks for the next 6 months. Both the clinical and radiographic assessments included the presence of tenderness at the fracture site, abnormal mobility, presence of infection, pain on movement of the knee and ankle joints and the anteroposterior and lateral radiographs of the leg. The results were assessed using Johner and Wruh's criteria in Annexure B.

Statistical analysis: The results were assessed using Johner and Wruh's criteria in Annexure B

\section{Results}

In the present study, 30 patients with distal tibia extraarticular fractures with AO type 43 A were selected and 15 of them were operated with closed interlocking intramedullary nailing and remaining 15 with a locking plate by MIPO. The average age in group A (ILN) was 40 years while in group B (MIPO) was about 43 years. The ' $t$ ' test results reveal that there was no significant difference $(p>0.05)$ between the age distribution among the two groups. The sex distribution in group A revealed about $87 \%$ were males and $13 \%$ were females, while in group B about $90 \%$ of the patients were males and about $7 \%$ of the patients were females. The Chisquare test reveals no significant association $(p>0.05)$ between the groups.

More than $60 \%$ of the patients in both the groups met with a road traffic accident while about $33 \%$ of the patients stated falls as their mode of injury. About $7 \%$ of the patients in group B suffered from the fracture due to assault.

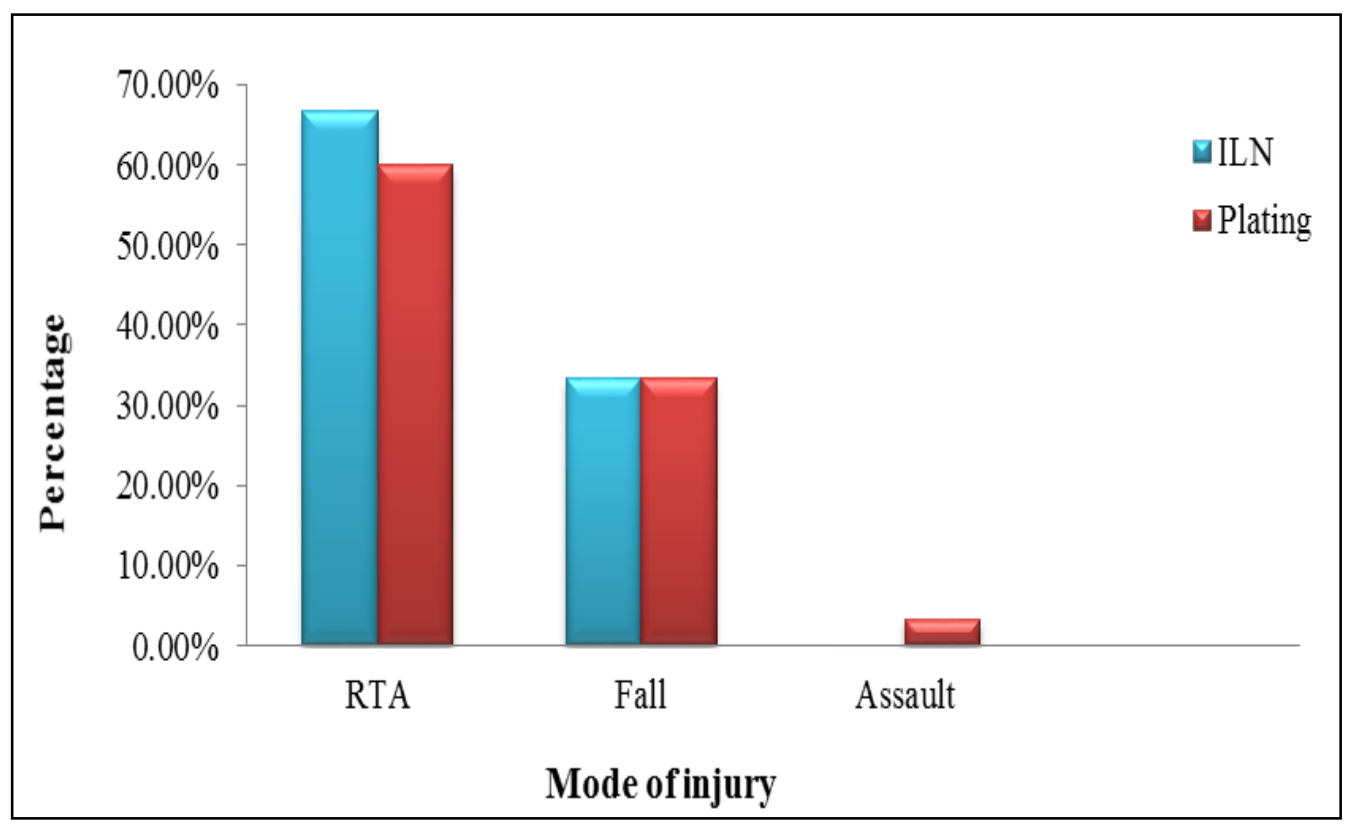

Graph 1: Mode of injury

About $53 \%$ of the patients with ILN and $60 \%$ of the patients with plating had left side injury. The same percentage (46.6\%) of patients from both the groups had 43A.1 type of fracture. About $33 \%$ from group A and $20 \%$ of patients of group B had 43 A.2 fractures and about 20 of patients from group A and 335 from group B had 43A.3 factors. The $p$ value $(>0.05)$ revealed no statistically significant difference between the groups. The average duration of the condition from trauma to surgery for the treatment of distal tibia fractures in the present study was 6.83 days.

In the interlocking group the average time from trauma to surgery was 6.53 days, whereas in the plating group the average time was 6.47 days. The $p$ value showed that the difference was not statistically significant. About $60 \%$ of the patients with ILN and $93.33 \%$ of the patients with plating had fracture of the fibula. The fixation of fibula in patients with distal tibia-fibula was performed in $26.66 \%$ in the interlocking group, while in the plating group, the fixation of fibula was done in $73.33 \%$ of the patients. The average duration of surgery in the interlocking group was $57.33 \mathrm{~min}$ and in the plating group was $70.36 \mathrm{~min}$. The $\mathrm{p}$ value $(<0.05)$ revealed the difference to be statistically significant. 


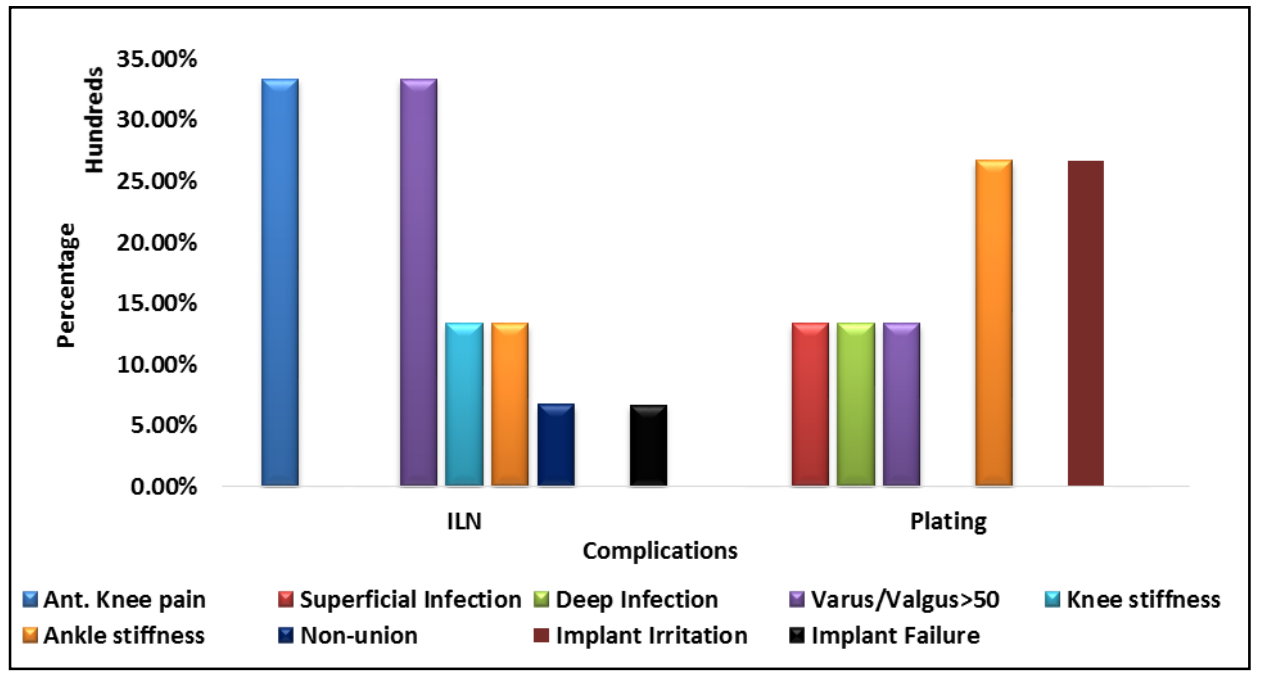

Graph 2: Complications

The average time for total weight bearing from surgery was 9.53 weeks in ILN, while in the plating group it was 13.29 weeks. This indicates that the ILN group started early total weight bearing than the plating group. The $\mathrm{p}$ value showed the difference to be statistically significant. About $26.66 \%$ of the patients from group B (plating group) complained of complications such as ankle stiffness and implant irritation.
About $13.33 \%$ of the patients from the same group complained of superficial infection, deep infection and angulation varus/valgus $>5^{0}$. About $33.33 \%$ of the patients who had undergone ILN surgery complained of anterior knee pain and angulation varus/valgus $>5^{\circ}$. About $133.33 \%$ had ankle stiffness, $6.66 \%$ had non-union and implant failure, and $3.33 \%$ had knee stiffness.

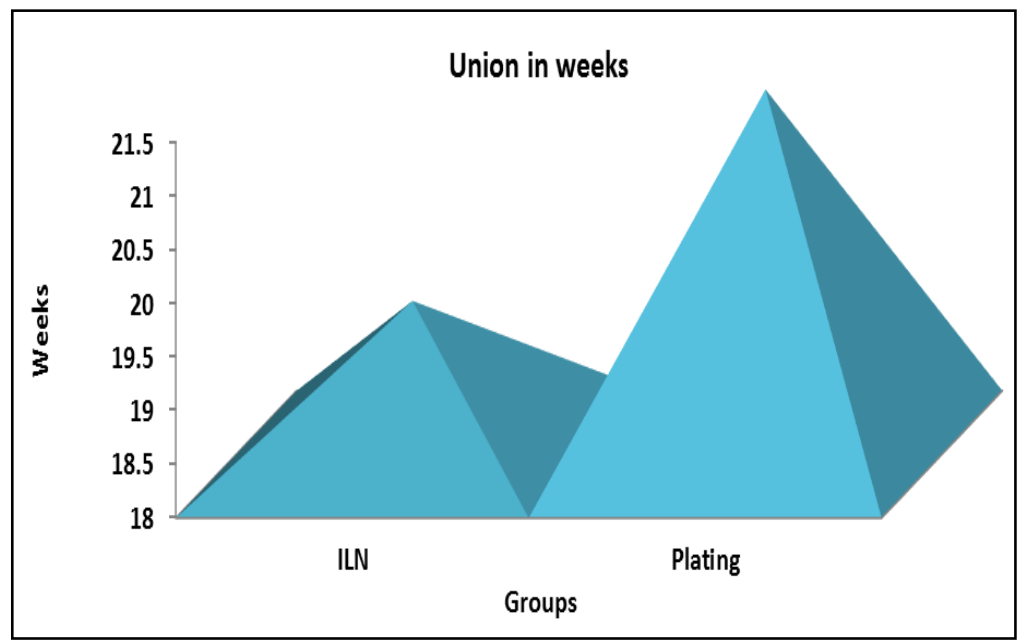

Graph 3: Union time

The average time for the union of distal tibia fractures was 20.41 weeks. The healing was faster in the patients with ILN surgery (19.43 weeks) than the patients undergone plating
(21.40 weeks). The difference was not statistically significant $(p>0.05)$.

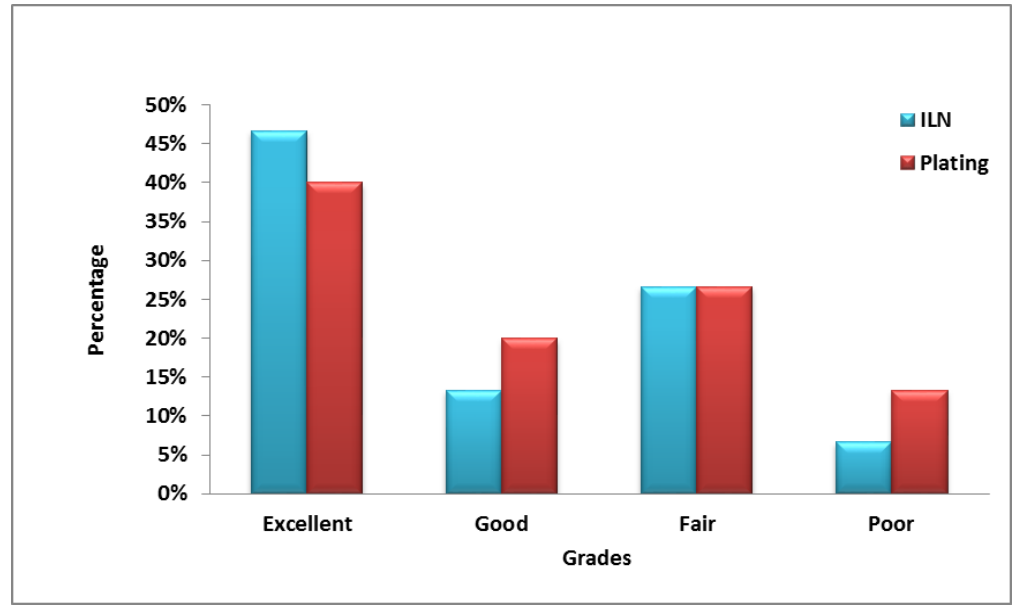

Graph 4: Results according to Johner and Wruh's score $\sim 148 \sim$ 
According to the Johner and Wruh's score, 53.33\% of the patients who had undergone ILN reported excellent recovery, $13.33 \%$ were good, 26.66 were fair and $6.66 \%$ showed poor recovery. Among the patients who had undergone plating, $40 \%$ were excellent, $20 \%$ were good, $26.66 \%$ were fair and $13.33 \%$ showed poor recovery.

\section{Discussion}

The distal tibial fractures are one of the most challenging fractures experienced by orthopedicians. It is due to its subcutaneous location, minimal vascularity and reduced muscular cover. There are chances of complications such as non-union, delayed union, infection and dehiscence, The two methods MIPO and IMLN are popularly used to treat distal tibial fractures but there procedures are also associated with various complications. The present study has been conducted to compare the clinical and radiological outcomes in extraarticular distal tibia fractures. In the present study, 30 patients with extra-articular distal tibia were enrolled for the study and were studied from March 2014 to March 2015. The follow up of the patients ranged from 6 months to 22 months. The results were observed and compared to various similar studies.

In a study conducted by Daolagupu, Mudgal, Agarwala, and Dutta (2017), a total of 42 patients participated in the study with 21 each in the IMLN group and the MIPO group.The mean age was 37.14 years which ranged from 19 to 59 years. The mean age of the patients in the IMLN group ranged from 19 to 50 years with the mean age of $35.19 \pm 9.22$ years. The mean age of the patients in MIPO group was $39.09 \pm 10.13$ years, which ranged from 20 to 59 years. Further, 32 males and 10 females participated in the study with $57.14 \%$ with the right tibia affected (2). The results were similar to the present study where the mean age of the patients in ILN and MIPO groups were 40 years and 43 years respectively. Additionally, 27 males and 3 females participated in the study.

Similar to the present study, the study conducted by Somashekhar, Ranganath< Shah, Murthy and Girish (2017) revealed that the $90 \%$ of the patients in the ILN group met with a road traffic accident and $10 \%$ experienced a fall. In the MIPO group, 50\% had a road traffic accident, $20 \%$ each had self-fall and fall from height, and $10 \%$ had a sports injury. Further, in $80 \%$ and $40 \%$ of patients with ILN and MIPO surgery, the duration of the surgery was 61 to 90 minutes respectively, which is similar to the results of the present study ${ }^{[3]}$.

In the study conducted by Kawalkar and Badole (2018), 87\% and $56 \%$ of the patients with ILN and MIPO surgery respectively, had $43 \mathrm{~A} 1$ type of fracture and $74 \%$ and $66 \%$ had fibula fracture respectively (4). The present study also showed that a high $(46.6 \%)$ number of fractures were 43A1 type in both the groups.

In the study conducted by Ali, Bhat, Bangroo, Muzaffar, Bhat, Dhanda and Maqsood (2014), it was observed that from the group I (ILN) and group II (MIPO), $6.67 \%$ and $20 \%$ had mal-union, $13.33 \%$ and $15 \%$ had delayed union and $13.33 \%$ and $3.33 \%$ had ankle stiffness respectively. Superficial infection was observed in $6.67 \%$ of the patients in both the groups ${ }^{[5]}$. In the present study, both deep and superficial infections were evident in the MIPO group. Nonunion with implant failure was observed in one patient and knee stiffness in $13.33 \%$ of the patients in the ILN group

\section{Conclusion}

The results of the present study conclude that both closed interlocking intramedullary nailing and locking plating by MIPO are equally safe and effective for the management of extra-articular distal tibia fracture. But, it is also observed that interlocking nailing is better in terms of early total weight bearing, fast union, and decreased risks of complications. Further it is also a cost effective and alternative method to MIPO for such kind of treatment.

\section{Reference}

1. Kawalkar AC, Badole CM. Distal tibia metaphyseal fractures: Which is better, intra-medullary nailing or minimally invasive plate osteosynthesis? Journal of Orthopaedics, Trauma and Rehabilitation. 2018, 24.

2. Shrestha D, Acharya B, Shrestha P. Minimally invasive plate osteosynthesis with locking compression plate for distal diametaphyseal tibia fracture. Kathmandu University Medical Journal. 2011; 34(2):62-68.

3. Im GI, Suk-Kee. Distal Metaphyseal Fractures of Tibia: A Prospective Randomized Trial of Closed Reduction and Intramedullary Nail Versus Open Reduction and Plate and Screws Fixation. Journal of Trauma-Injury Infection \& Critical Care. 2005; 59(5):1219-1223.

4. Janssen KW, Biert J, Kampen Av. Treatment of distal tibial fractures: plate versus nail, A retrospective outcome analysis of matched pairs of patients. International Orthopaedics (SICOT). 2007; 31:709-714.

5. Vallier HA, Toan LT, A. Radiographic and Clinical Comparisons of Distal Tibia Shaft Fractures (4 to $11 \mathrm{~cm}$ Proximal to the Plafond): Plating Versus Intramedullary Nailing. Journal of Orthopaedic Trauma. 2008; 22(5):307-311.

6. Daolagupu AK, Mudgal A, Agarwala V, Dutta KK. A comparative study of intramedullary interlocking nailing and minimally invasive plate osteosynthesis in extra articular distal tibial fractures. Indian Journal of Orthopedics. 2017; 51(3):292-298.

7. Ali N, Bhat A, Bangroo FA, Muzzafar K, Bhat SA, Dhanda MS et al. Treatment of Extra-Articular Distal Tibial Fractures: Minimally Invasive Percutaneous Plate Osteosynthesis Versus Intramedullary Nailing. Trauma Monthly. 2014; 22(4).

8. Kawalkar AC, Badole CM. Distal tibia metaphyseal fractures: Which is better, intra-medullary nailing or minimally invasive plate osteosynthesis? Journal of Orthopaedics, Trauma and Rehabilitation. 2018; 24:6671.

9. JTA. Bone plates, chapter 4 in "The elements of fracture fixation". 2nd ed. 2011.

10. Somashekhar, Ranganath, Shah BM, Murthy JS, SG. A comparative study of functional outcome of distal tibial extra-articular faracture fixed with intramedullary interlocking nail versus locking compression plate. International Journal of Orthopaedics Sciences. 2017; 3(2):80-85. 